\title{
Implications of the Pattern of Disease Recurrence on Survival Following Pancreatectomy for Pancreatic Ductal Adenocarcinoma
}

\author{
Vincent P. Groot, MD ${ }^{1,2}$, Georgios Gemenetzis, MD ${ }^{1}$, Alex B. Blair, MD ${ }^{1}$, Ding Ding, MD, \\ MS ${ }^{1}$, Ammar A. Javed, MD¹, Richard A. Burkhart, MD ${ }^{1}$, Jun Yu, MD, PhD ${ }^{1}$, Inne H. Borel \\ Rinkes, MD, PhD², I. Quintus Molenaar, MD, PhD², John L. Cameron, MD, FACS ${ }^{1}$, Elliot K. \\ Fishman, MD ${ }^{3}$, Ralph H. Hruban, MD ${ }^{4}$, Matthew J. Weiss, MD, FACS ${ }^{1}$, Christopher L. \\ Wolfgang, MD, PhD, FACS ${ }^{1}$, and Jin He, MD, PhD, FACS ${ }^{1}$
}

\begin{abstract}
${ }^{1}$ Department of Surgery, The Sol Goldman Pancreatic Cancer Research Center, The Johns Hopkins University School of Medicine, Baltimore, MD ${ }^{2}$ Department of Surgery, UMC Utrecht Cancer Center, University Medical Center Utrecht, Utrecht, The Netherlands ${ }^{3}$ Department of Radiology, The Sol Goldman Pancreatic Cancer Research Center, The Johns, Hopkins University School of Medicine, Baltimore, MD ${ }^{4}$ Department of Pathology, The Sol Goldman Pancreatic Cancer Research Center, The Johns Hopkins University School of Medicine, Baltimore, MD
\end{abstract}

\section{Abstract}

Background.-After radical resection of pancreatic ductal adenocarcinoma (PDAC), approximately $80 \%$ of patients will develop disease recurrence. It remains unclear to what extent the location of recurrence carries prognostic significance. Additionally, stratifying the pattern of recurrence may lead to a deeper understanding of the heterogeneous biological behavior of PDAC.

Objective.-The aim of this study was to characterize the relationship of recurrence patterns with survival in patients with resected PDAC.

\begin{abstract}
Methods.-This single-center cohort study included patients undergoing pancreatectomy at the Johns Hopkins Hospital between 2000 and 2013. Exclusion criteria were neoadjuvant therapy and incomplete follow-up. Sites of first recurrence were stratified into five groups and survival outcomes were estimated using Kaplan-Meier curves. The association of specific recurrence locations with overall survival (OS) was analyzed using Cox proportional-hazards models with and without landmark analysis.
\end{abstract}

Results._Accurate follow-up data were available for 877 patients, 662 (75.5\%) of whom had documented recurrence at last follow-up. Patients with multiple-site ( $n=227,4.7$ months) or liveronly recurrence ( $n=166,7.2$ months) had significantly worse median survival after recurrence

J. He, MD, PhD, FACS, jhe11@jhmi.edu.

DISCLOSURES Vincent P. Groot, Georgios Gemenetzis, Alex B. Blair, Ding Ding, Ammar A. Javed, Richard A. Burkhart, Jun Yu, Inne H. Borel Rinkes, I. Quintus Molenaar, John L. Cameron, Elliot K. Fishman, Ralph H. Hruban, Matthew J. Weiss, Christopher L. Wolfgang, and Jin He have no financial disclosures or conflicts of interest to declare.

Electronic supplementary material The online version of this article (https://doi.org/10.1245/s10434-018-6558-7) contains supplementary material, which is available to authorized users. 
when compared with lung- $(n=93)$ or local-only $(n=158)$ recurrence (15.4 and 9.7 months, respectively). On multivariable analysis, the unique recurrence patterns had variable predictive values for OS. Landmark analyses, with landmarks set at 12, 18, and 24 months, confirmed these findings.

Conclusions.-This study demonstrates that specific patterns of PDAC recurrence result in different survival outcomes. Furthermore, distinct first recurrence locations have unique independent predictive values for OS, which could help with prognostic stratification and decisions regarding treatment after the diagnosis of recurrence.

Pancreatic ductal adenocarcinoma (PDAC) is projected to become the second most common cause of cancer-related death in the US in the next 15 years and continues to be one of the most treatment-resistant malignancies. ${ }^{1}$ Radical resection of localized PDAC provides the best chance for long-term survival, and many studies have investigated prognostic factors for predicting survival outcomes after pancreatectomy. ${ }^{2-4}$

Unfortunately, even after successful radical resection of PDAC, up to $80 \%$ of patients will develop disease recurrence ${ }^{5-9}$ A recent study from our institution found that the time to recurrence differed for distinct postoperative recurrence locations; $;{ }^{10}$ however, the relationship of these specific PDAC recurrence locations with overall survival (OS) remains poorly described. Knowledge on the prognostic impact of distinct recurrence sites might help clinicians with prognostic stratification and provide a better understanding of the heterogeneous biological behavior of PDAC.

The aim of this study was therefore twofold. First, to characterize the association of patterns of disease recurrence with survival after recurrence (SAR), and, second, to investigate the predictive value of specific recurrence locations on OS while adjusting for known prognostic clinicopathologic variables. To avoid bias in examining OS by recurrence location, careful statistical analysis was performed using proportional hazard models, with and without landmark analysis, in a large cohort of patients who underwent surgery for primary resectable PDAC in the head of the gland.

\section{METHODS}

\section{Patient Selection}

This single-center cohort included patients who underwent a standard pancreatoduodenectomy, pyloruspreserving pancreatoduodenectomy, or total pancreatectomy for PDAC at the Johns Hopkins Hospital between 2000 and 2013. Only patients who underwent upfront resection of resectable or borderline resectable PDAC in the head of the pancreas were included. Resectability and staging were generally discussed in a multidisciplinary setting and based on preoperative pancreatic protocol computed tomopraphy (CT) imaging. Patients were staged with resectable disease (stage I) when there was no arterial and/or venous involvement, and with borderline resectable disease (stage II) when there was limited arterial and/or venous involvement allowing for safe and complete resection with or without vascular reconstruction. Apart from neoadjuvant therapy, other exclusion criteria were (1) synchronous distant metastases at the time of surgery; (2) macroscopic margin-positive resection $\left(R_{2}\right)$; or (3) 90-day surgery-related mortality. Patients 
with incomplete follow-up data due to loss to follow-up or follow-up performed at other institutions were also excluded. Institutional Review Board approval was obtained for this retrospective study.

\section{Demographic, Clinicopathological, and Treatment Characteristics}

Demographic and clinical variables were extracted from a prospective institutional database. Perioperative complications were reported according to the Clavien- Dindo classification. ${ }^{11}$ The resection margin $(R)$ was defined as $\mathrm{R}_{0}$ when the distance of carcinoma cells to the closest resection margin was $>1 \mathrm{~mm}$, and $R_{1}$ when the distance was $\geq 1 \mathrm{~mm}$. If their performance status allowed, patients with recurrence were further treated with systemic therapy. In highly selected patients, localized treatments such as stereotactic body radiation therapy for isolated local recurrence, or metastasectomy for stable isolated lung recurrence, were sporadically performed.

\section{Follow-Up and Recurrence Definitions}

Follow-up methodology and definitions of disease recurrence after pancreatectomy at our institution have been described previously. ${ }^{10}$ The first location of disease recurrence was documented based on either radiological or histological evidence; however, confirmation of recurrence with a biopsy was not routinely performed. Recurrence locations were stratified into five mutually exclusive categories: 'local-only', 'liver-only', 'lung-only', 'multiplesite' and 'other'. Multiple-site recurrence included patients with local and distant recurrence, recurrence in multiple distant sites (i.e. lung and liver) and/or carcinomatosis.

\section{Survival Outcomes}

Recurrence-free survival was defined as the time interval between the date of surgery and the date of recurrence, while OS was defined as the time from the date of surgery to either death from any cause or last follow-up. Cause of death was retrieved from our institutional database, cross-referenced, and supplemented with data obtained from the National Death Index of the Centers for Disease Control and Prevention. Disease-specific survival was defined as the time from the date of operation to either death due to PDAC or last follow-up, with censoring occurring for patients who died of any other cause. Lastly, SAR was defined as the time from recurrence to either death or last follow-up.

\section{Statistical Analysis}

Kaplan-Meier curves and the log-rank test were used to estimate median survival outcomes with a corresponding $95 \%$ confidence interval $(\mathrm{CI})$, and to compare between subgroups. To establish whether recurrence location as a time-dependent covariate has a prognostic effect on the OS of patients with resected PDAC, while adjusting for other clinicopathologic variables, two distinct multivariable Cox proportional-hazards regression models were used.

First, a conventional Cox model was created in which recurrence locations were considered as fixed covariates. However, since specific recurrence locations have different recurrencefree intervals, ${ }^{10}$ this introduces a potential bias when assessing OS directly. More specifically, local or lung recurrence generally occur relatively late after surgery, which might result in a 'lead time' or 'guarantee time' bias towards improved OS when compared 
with multiple-site or liver-only recurrence, which tend to arise after a shorter recurrence-free interval.

A statistical technique to account for this type of potential bias is a landmark analysis (see also the electronic supplementary Methods). ${ }^{12-15}$ A landmark time of 12 months was chosen, ${ }^{16}$ and a multivariable Cox model was then conducted with survival from the landmark. To examine whether this choice of landmark affected either strength or direction of the found relationships between recurrence locations and survival, similar analyses were performed with landmarks set at 18 and 24 months.

A stepwise backward approach using Akaike information criterion (AIC) was used to select covariates for the multivariable models. Internal validation and optimism for the multivariable model with landmark analysis set at 12 months was performed with 200 times bootstrap. The proportional hazards assumption of the Cox models was checked using Schoenfeld residuals. A two-tailed $P$ value $<0.05$ was considered to indicate statistical significance. Statistical analysis was performed using SPSS version 25.0 (IBM Corporation, Armonk, NY, USA) and R version 3.3.3 (R Foundation, Vienna, Austria).

\section{RESULTS}

A total of 1325 patients who underwent upfront pancreatectomy for histologically confirmed PDAC were included in our study. Excluded were 427 patients (32.2\%) with incomplete follow-up data due to loss to follow-up or follow-up performed at other institutions, as well as a further 21 patients (1.6\%) who died within 90 days after surgery. Consequently, a final cohort of 877 patients with accurate recurrence and survival data was established.

\section{Cohort Characteristics}

Detailed demographic, clinicopathologic, and treatment characteristics of the included patients are summarized in Table 1. Median follow-up for all patients was 23.6 months (95\% CI 21.8-25.3). At last follow-up, disease recurrence was recorded in 662 patients (75.5\%). A total of 215 patients $(24.5 \%$ ) had no sign of recurrence after a median follow-up of 46.3 months (95\% CI 36.4-54.3). The most common manifestation was multiplesite recurrence ( $n=227,34.3 \%)$, followed by liver-only $(n=166,25.1 \%)$, local-only $(n=158,23.9 \%)$, and lungonly $(n=93,14.0 \%)$ recurrence. Other recurrence sites $(n=18,2.7 \%)$ included osseous structures, the brain, supraclavicular lymph nodes, groin, thigh muscle, and the skin.

\section{Cause of Death}

At last follow-up, 186 patients (21.2\%) were alive after a median follow-up of 56.4 months (95\% CI 45.0-67.8). Of the 691 patients who died, $81.8 \%$ died of PDAC $(n=565)$. Other causes included cardiovascular disease $(n=28,4.1 \%)$, other primary cancers $(n=26,3.8 \%)$, infectious disease $(n=13,1.9 \%)$, and lung disease causing respiratory failure $(n=9,1.3 \%)$. Several other less common causes, including but not limited to accidents, suicide, or drug overdose, amounted to $2.3 \%(n=16)$ of the reported deaths.

Thirty-four patients (4.9\%) died of unknown cause but without documented recurrence on the most recent imaging. Of the 140 patients still alive $<5$ years after surgery, 50 (35.7\%) 
went on to die during later follow-up, of whom $23(46.0 \%)$ were due to causes other than PDAC. In patients who survived 55 years, the ratio of death due to other causes $(103 / 641$, $16.1 \%)$ was significantly lower $(P<0.001)$. Furthermore, $45 / 147$ patients $(30.6 \%)$ who were $>75$ years of age and died, passed away due to other causes, compared with $81 / 544$ patients $(14.9 \%)$ who were $\leq 75$ years of age at the time of surgery $(P<0.001)$. Rates of death due to PDAC did not differ significantly among the different recurrence patterns.

\section{Survival Outcomes}

Median survival outcomes for all patients and per different recurrence location are presented in Table 2. Patients with multiple-site or liver-only recurrence had a limited median SAR of 4.7 months (95\% CI 3.8-5.1) and 7.2 months (95\% CI 6.0-8.5), respectively, which were both significantly worse when compared with survival after local- or lung-only recurrence (Fig. 1). Patients with lungonly recurrence had a median SAR of 15.4 months (95\% CI 10.919.9), significantly longer than the second longest median SAR of patients with local-only recurrence (9.7 months, 95\% CI 7.5-12.0, $P=0.001)$. Further analysis showed that patients who recurred early had relatively limited survival after their recurrence. Overall, 338 patients with a recurrence-free survival of $\leq 12$ months had a median SAR of 6.0 months (95\% CI 5.2-6.8), significantly shorter than the 10.0 months (95\% CI 9.1-12.1) for the 324 patients who recurred after more than 12 months $(\mathrm{P}<0.001)$.

Median OS of 662 patients with recurrence (21.1 months, 95\% CI 19.0-23.2) was considerably shorter when compared with the 83.9 months (95\% CI 59.3-108.6) found for 215 patients without recurrence $(P<0.001)$ [Table 2]. Pairwise comparison per specific recurrence patterns revealed that median OS varied significantly based on recurrence location (Fig. 2). Lung-only, local-only and other recurrence were all associated with considerably longer median OS when compared with multiple-site or liver-only recurrence.

\section{Multivariable Analysis}

In the multivariable Cox regression model with recurrence locations as fixed covariates, all recurrence patterns were significantly associated with worse OS when compared with nonrecurrent patients (Table 3). Multiple-site and liver-only recurrence both had hazard ratios (HRs) of more than 4, indicative of their strong independent association with a limited OS. While local-only, lung-only, and other recurrence were also significantly correlated with decreased OS, their HRs of $<2.5$ showed a weaker relationship. Furthermore, adjuvant therapy (HR $0.61,95 \%$ CI $0.52-0.72, P<0.001$ ) was independently associated with prolonged OS.

To adjust for potential bias in the first model, multivariable Cox models for OS using landmark analyses were additionally performed. At the chosen landmark time of 12 months, 200 patients (22.8\%) had died or were lost to follow-up. As a result, 677 patients were available for analysis, 180 (26.6\%) of whom had recurrence at that time. Similar to the first Cox model with fixed covariates, the landmark analysis established that all recurrence locations were associated with worse OS, with the strongest relationship found for multiplesite and liver-only recurrence (Table 4). The model with landmark at 12 months had a Cstatistic of 0.696. Since internal validation with 200 times bootstrap showed an optimism of 
0.009 , the optimism-corrected performance of the model was 0.687. Further multivariable analyses using landmarks set at 18 and 24 months found comparable relationships between the different recurrence patterns and OS (electronic supplementary Tables 1 and 2).

\section{DISCUSSION}

This study demonstrates that OS and survival after PDAC recurrence differ based on the site of first recurrence. Specifically, the current study found that patients with multiple-site recurrence had a limited median SAR of $<5$ months, while patients who developed pulmonary metastases had a median survival of $>15$ months after first diagnosis of recurrence. Furthermore, patients with isolated lung or local recurrence had a considerably prolonged median OS. Lastly, using two different techniques of multivariable Cox regression analysis, specific recurrence patterns were shown to have distinct independent predictive values for the prognosis of OS.

A prior study focusing on the timing and predictors of PDAC recurrence, consisting of a subset of the present cohort, showed that specific recurrence locations occur after distinct recurrence-free intervals. ${ }^{10}$ Furthermore, different recurrence locations were shown to have unique predictive factors. The current findings that show that specific recurrence locations have different post-pancreatectomy and post-recurrence survival further supplement and expand on these previous findings, and continue to support the hypothesis that unique biological behaviors exist among PDACs.

Relatively few studies have focused on the impact of the initial relapse pattern on postpancreatectomy survival outcomes. ${ }^{9}$ Moreover, associations observed in earlier studies vary considerably, possibly due to limited sample size and length of follow-up. For instance, van den Broeck et al. ${ }^{6}$ found no association between recurrence location and survival, while Tani et al. ${ }^{17}$ found similar median OS for patients with and without initial liver metastases. However, other studies did find significant correlations between patterns of recurrence and survival outcomes. When considering SAR, Sperti et al. ${ }^{5}$ showed that median survival time from the detection of recurrence until death was 7 months for patients with isolated local recurrence versus 3 months for patients with multiple-site or liveronly recurrence $(P<0.05)$. Similar to our results, Zhang and colleagues ${ }^{18}$ found that patients with locoregional recurrence (10.4 months) had significantly improved SAR when compared with those with isolated distant (5.5 months, $P=0.002)$ or multiple-site (5.8 months, $P=0.044)$ recurrence.

The current study demonstrates that recurrence at multiple sites or in the liver leads to a relatively limited OS of 18 and 15 months, respectively. In addition, both of these manifestations of recurrent PDAC were strong independent predictors for decreased OS in all multivariable models. Similarly, in a study of 209 patients, Suenaga et al. ${ }^{8}$ reported that patients with hepatic relapse had significantly shorter OS than those without hepatic relapse (12.9 months vs. 18.4 months, $P<0.001$ ), and that local recurrence was associated with a more favorable prognosis than recurrences at other sites (18.4 months vs. 14.7 months, $P=$ $0.010)$. 
This study aimed to accurately identify the correlation between five unique PDAC recurrence patterns and subsequent SAR. Recurrence of PDAC following resection imposes a difficult choice when considering the pursuit of additional treatment. The presented results provide prognostic data to further assist patient and physician alike when discussing challenging clinical choices and the balance of quality of life with further treatment options. Patients with isolated local or pulmonary recurrence were found to have relatively favorable survival outcomes. The favorable prognosis for patients with isolated local recurrence might stem from the fact that local recurrence can originate from microscopic residual disease in the remnant pancreas that has not gone through the process of hematogenous dissemination. This seemingly less-hostile tumor biology and slower-growing propensity might thus warrant additional local treatment. Initial experiences at our institution with stereotactic body radiation therapy for isolated local recurrence have shown encouraging results, especially in patients who had a relatively long recurrencefree interval. ${ }^{19,20}$

Patients with pulmonary metastases had both the longest median SAR (15 months) and OS (40 months), possibly representing another unique clinical and biological subtype of PDAC in which more aggressive and/or localized additional therapy might be justified. A study from our institution, for example, showed improved median OS of 51 months after pulmonary metastasectomy in highly selected patients. ${ }^{21}$ Although the present literature is limited, several other studies have reported on the benefit of either surgical resection or stereotactic radiosurgery for lung metastases, highlighting increased interest in additional treatment options. $^{22-26}$

The records of specific causes of death revealed that up to $18 \%$ of patients died from causes other than PDAC after surgical resection. Interestingly, more than 35\% of patients who died after being recurrence-free for 5 years did so due to other causes. In addition, approximately $30 \%$ of deaths among patients older than 75 years at the time of operation were attributed to other diseases. However, these results should be interpreted with caution as several of the causes of death that were recorded, such as cholangitis, liver abscesses, or even suicide, might have been indirectly related to PDAC or the treatment thereof. Similarly, the taxing aftermath of pancreatic surgery and subsequent adjuvant therapy might have exacerbated existing comorbidities or weakened the immune system of older patients, resulting in more deaths credited to other causes such as cardiovascular events and infectious diseases.

Our study has several other important limitations worthy of mentioning. First, our database merely captured the recurrence location, without commenting on the extent of recurrent disease. Second, recurrence location was generally based on radiographic findings without additional tissue confirmation. This might have influenced the identified rates of recurrence, as, for instance, benign pulmonary nodules are seen commonly and are often difficult to differentiate from true pulmonary metastases. ${ }^{27}$ Third, many patients choose to receive further therapy from local institutions, limiting the accuracy of data on type, dosage, and frequency of therapy for recurrence that would have potentially exposed correlations not currently established. Lastly, the number of patients who were lost to follow-up was noteworthy, potentially limiting the generalizability of our findings. On the other hand, a significant strength of this study was the use of a prospective database from a large tertiary referral center for patients with PDAC. Furthermore, additional multivariable models using 
landmark analysis were performed to adjust for potential bias caused by the distinct timing of different recurrence patterns.

\section{CONCLUSIONS}

This study demonstrates that specific patterns of PDAC recurrence are associated with different survival outcomes. Furthermore, distinct locations of the first recurrence were shown to have unique independent predictive values for OS, which could help with prognostic stratification and decisions regarding treatment after the diagnosis of recurrence. In support of earlier findings, these results continue to suggest that ductal adenocarcinoma of the pancreas is a highly heterogenic disease and future studies should focus on developing patient-tailored multimodality treatment approaches that take into account these diverse biological behaviors.

\section{Supplementary Material}

Refer to Web version on PubMed Central for supplementary material.

\section{ACKNOWLEDGEMENT}

The authors would like to thank the Foundation "De Drie Lichten”, Prins Bernhard Cultuurfonds, VSBfonds, Prof. Michae"1-van Vloten Fonds, Nijbakker-Morra Foundation, and the Living With Hope Foundation (all from The Netherlands) for funding this study in the form of grants for a research fellowship by VPG.

\section{REFERENCES}

1. Rahib L, Smith BD, Aizenberg R, Rosenzweig AB, Fleshman JM, Matrisian LM. Projecting cancer incidence and deaths to 2030: the unexpected burden of thyroid, liver, and pancreas cancers in the United States. Cancer Res. 2014;74:2913-21. [PubMed: 24840647]

2. Riall TS, Cameron JL, Lillemoe KD, Winter JM, Campbell KA,Hruban RH, et al. Resected periampullary adenocarcinoma: 5-year survivors and their 6- to 10-year follow-up. Surgery. 2006;140:764-72. [PubMed: 17084719]

3. Cameron JL, Riall TS, Coleman J, Belcher KA. One thousandconsecutive pancreaticoduodenectomies. Ann Surg. 2006;244:10-15. [PubMed: 16794383]

4. Cameron JL, He J. Two thousand consecutive pancreaticoduodenectomies. J Am Coll Surg. 2015;220:530-6. [PubMed: 25724606]

5. Sperti C, Pasquali C, Piccoli A, Pedrazzoli S. Recurrence afterresection for ductal adenocarcinoma of the pancreas. World J Surg. 1997;21:195-200. [PubMed: 8995078]

6. Van den Broeck A, Sergeant G, Ectors N, Van Steenbergen W,Aerts R, Topal B. Patterns of recurrence after curative resection of pancreatic ductal adenocarcinoma. Eur J Surg Oncol. 2009;35:600-4. [PubMed: 19131205]

7. Hernandez JM, Morton CA, Al-Saadi S, Villadolid D, Cooper J,Bowers C, et al. The natural history of resected pancreatic cancer without adjuvant chemotherapy. Am Surg. 2010;76:480-5. [PubMed: 20506876]

8. Suenaga M, Fujii T, Kanda M, Takami H, Okumura N, Inokawa Y, et al. Pattern of first recurrent lesions in pancreatic cancer: hepatic relapse is associated with dismal prognosis and portal vein invasion. Hepatogastroenterology. 2014;61:1756-61. [PubMed: 25436375]

9. Groot VP, Daamen LA, Hagendoorn J, Borel Rinkes IHM, vanSantvoort HC, Molenaar IQ. Use of imaging during symptomatic follow-up after resection of pancreatic ductal adenocarcinoma. J Surg Res. 2018;221:152-60. [PubMed: 29229122] 
10. Groot VP, Rezaee N, Wu W, Cameron JL, Fishman EK, Hruban RH, et al. Patterns, timing, and predictors of recurrence following pancreatectomy for pancreatic ductal adenocarcinoma. Ann Surg. 2018;267:936-45. [PubMed: 28338509]

11. Clavien PA, Barkun J, de Oliveira ML, Vauthey JN, Dindo D,Schulick RD, et al. The ClavienDindo classification of surgical complications: five-year experience. Ann Surg. 2009;250:187-96. [PubMed: 19638912]

12. Anderson JR, Cain KC, Gelber RD. Analysis of survival by tumorresponse. J Clin Oncol. 1983;1:710-9. [PubMed: 6668489]

13. Weeden S, Grimer RJ, Cannon SR, Taminiau AH, Uscinska BM.The effect of local recurrence on survival in resected osteosarcoma. Eur J Cancer. 2001;37:39-46. [PubMed: 11165128]

14. Dafni U Landmark analysis at the 25-year landmark point. Circ Cardiovasc Qual Outcomes. 2011;4:363-71. [PubMed: 21586725]

15. Hallet J, Sa Cunha A, Adam R, Goe're’ D, Bachellier P, Azoulay D, et al. Factors influencing recurrence following initial hepatectomy for colorectal liver metastases. Br J Surg. 2016;103:1366-76. [PubMed: 27306949]

16. Groot VP, Gemenetzis G, Blair AB, Rivero-Soto RJ, Yu J, Javed AA, et al. Defining and predicting early recurrence in 957 Patients with resected pancreatic ductal adenocarcinoma. Ann Surg. Epub 2332018 10.1097/sla.0000000000002734.

17. Tani M, Kawai M, Miyazawa M, Hirono S, Ina S, Nishioka R,et al. Liver metastasis as an initial recurrence has no impact on the survival of patients with resectable pancreatic adenocarcinoma. Langenbecks Arch Surg. 2009;394:249-53. [PubMed: 18343944]

18. Zhang Y, Frampton AE, Kyriakides C, Bong JJ, Habib N,Ahmad R, et al. Loco-recurrence after resection for ductal adenocarcinoma of the pancreas: predictors and implications for adjuvant chemoradiotherapy. J Cancer Res Clin Oncol. 2012;138:1063-71. [PubMed: 22392075]

19. Groot VP, van Santvoort HC, Rombouts SJE, Hagendoorn J,Borel Rinkes IH, van Vulpen M, et al. Systematic review on the treatment of isolated local recurrence of pancreatic cancer after surgery; re-resection, chemoradiotherapy and SBRT. HPB (Oxford). 2017;19:83-92. [PubMed: 28065427]

20. Ryan JF, Groot VP, Rosati LM, Hacker-Prietz A, Narang AK,McNutt TR, et al. Stereotactic body radiation therapy for isolated local recurrence after surgical resection of pancreatic ductal adenocarcinoma appears to be safe and effective. Ann Surg Oncol. 2018;25:280-89. [PubMed: 29063299]

21. Arnaoutakis GJ, Rangachari D, Laheru DA, Iacobuzio-Donahue CA, Hruban RH, Herman JM, et al. Pulmonary resection for isolated pancreatic adenocarcinoma metastasis: an analysis of outcomes and survival. J Gastrointest Surg. 2011;15:1611-7. [PubMed: 21725701]

22. Downs-Canner S, Zenati M, Boone BA, Varley PR, Steve J,Hogg ME, et al. The indolent nature of pulmonary metastases from ductal adenocarcinoma of the pancreas. J Surg Oncol. 2015;112:80 85. [PubMed: 26153355]

23. Wangjam T, Zhang Z, Zhou XC, Lyer L, Faisal F, Soares KC,et al. Resected pancreatic ductal adenocarcinomas with recurrence limited in lung have a significantly better prognosis than those with other recurrence patterns. Oncotarget. 2015;6:36903-10. [PubMed: 26372811]

24. Lu F, Poruk KE, Weiss MJ. Surgery for oligometastasis of pancreatic cancer. Chin J Cancer Res. 2015;27:358-67. [PubMed: 26361405]

25. Yamashita K, Miyamoto A, Hama N, Asaoka T, Maeda S, Omiya H, et al. Survival impact of pulmonary metastasis as recurrence of pancreatic ductal adenocarcinoma. Dig Surg. 2015;32:46471. [PubMed: 26517228]

26. Zheng B, Ohuchida K, Yan Z, Okumura T, Ohtsuka T, Nakamura M. Primary recurrence in the lung is related to favorable prognosis in patients with pancreatic cancer and postoperative recurrence. World J Surg. 2017;41: 2858-66. [PubMed: 28634843]

27. Poruk KE, Kim Y, Cameron JL, He J, Eckhauser FE, Rezaee N, et al. What is the significance of indeterminate pulmonary nodules in patients undergoing resection for pancreatic adenocarcinoma? J Gastrointest Surg. 2015;19:841-47. [PubMed: 25595307] 


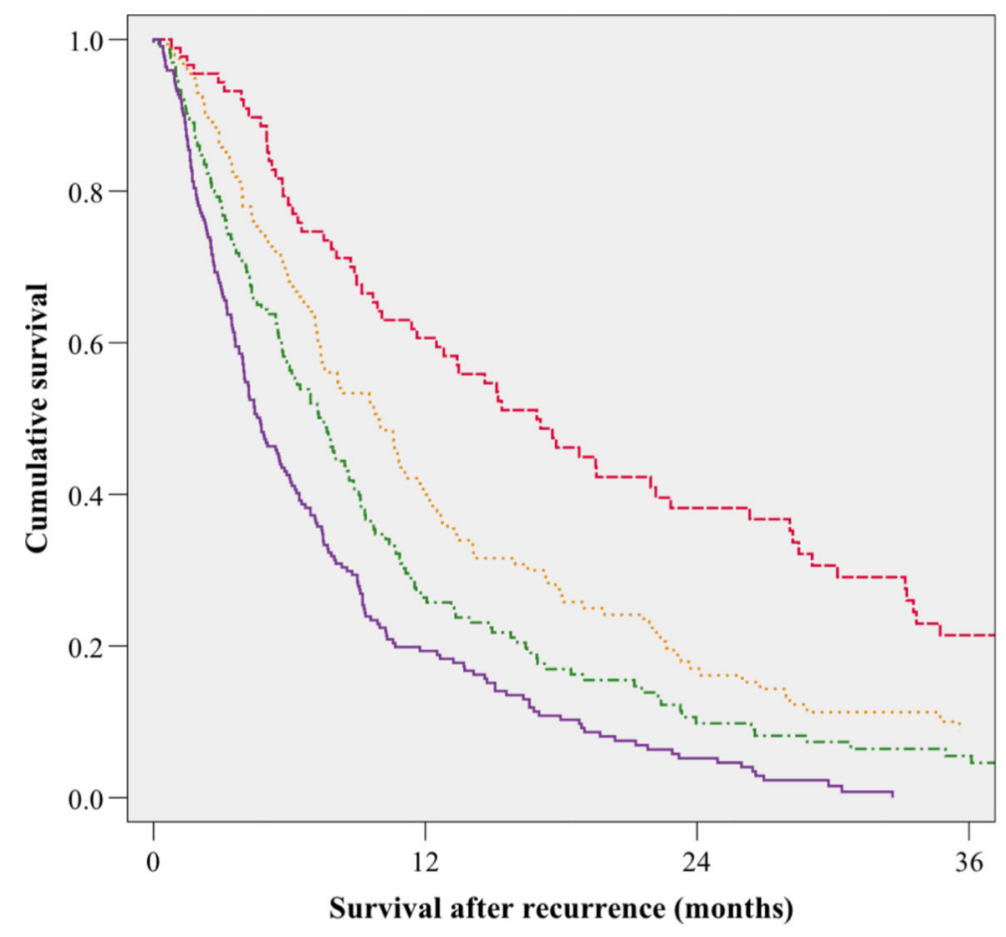

Location of First

Recurrence

Local-only

- Liver-only

ITLung-only

$\neg$ Multiple-site

\begin{tabular}{lcccccc}
\hline $\begin{array}{l}\text { Recurrence } \\
\text { pattern }\end{array}$ & $\begin{array}{l}\text { Lung- } \\
\text { only }\end{array}$ & $\begin{array}{l}\text { Local- } \\
\text { only }\end{array}$ & Other & $\begin{array}{l}\text { Multiple- } \\
\text { site }\end{array}$ & $\begin{array}{l}\text { Liver- } \\
\text { only }\end{array}$ & $\begin{array}{l}\text { Median SAR } \\
\text { months (95\%CI) }\end{array}$ \\
\hline $\begin{array}{l}\text { Lung-only } \\
(\boldsymbol{n}=\mathbf{9 3})\end{array}$ & $\mathbf{0 . 0 0 1}$ & $\mathbf{0 . 0 3 4}$ & $<\mathbf{0 . 0 0 1}$ & $<\mathbf{0 . 0 0 1}$ & $15.4(10.9-19.9)$ \\
\hline $\begin{array}{l}\text { Local-only } \\
(\boldsymbol{n}=\mathbf{1 5 8})\end{array}$ & $\mathbf{0 . 0 0 1}$ & & 0.559 & $<\mathbf{0 . 0 0 1}$ & $\mathbf{0 . 0 0 8}$ & $9.7(7.5-12.0)$ \\
\hline $\begin{array}{l}\text { Other } \\
(\boldsymbol{n}=\mathbf{1 8})\end{array}$ & $\mathbf{0 . 0 3 4}$ & 0.559 & & $\mathbf{0 . 0 4 1}$ & 0.389 & $8.0(1.8-14.3)$ \\
\hline $\begin{array}{l}\text { Multiple-site } \\
(\boldsymbol{n}=\mathbf{2 2 7})\end{array}$ & $<\mathbf{0 . 0 0 1}$ & $<\mathbf{0 . 0 0 1}$ & $\mathbf{0 . 0 4 1}$ & & $\mathbf{0 . 0 0 1}$ & $4.7(3.8-5.1)$ \\
\hline $\begin{array}{l}\text { Liver-0nly } \\
(\boldsymbol{n}=\mathbf{1 6 6})\end{array}$ & $<\mathbf{0 . 0 0 1}$ & $\mathbf{0 . 0 0 8}$ & 0.389 & $\mathbf{0 . 0 0 1}$ & & $7.2(6.0-8.5)$ \\
\hline
\end{tabular}

SAR, survival after recurrence; CI, confidence interval.

FIG. 1.

Kaplan-Meier curves showing SAR for different recurrence patterns with pairwise comparison of SAR using the log-rank test. For clarity, the curve for 'other' recurrence has been omitted 


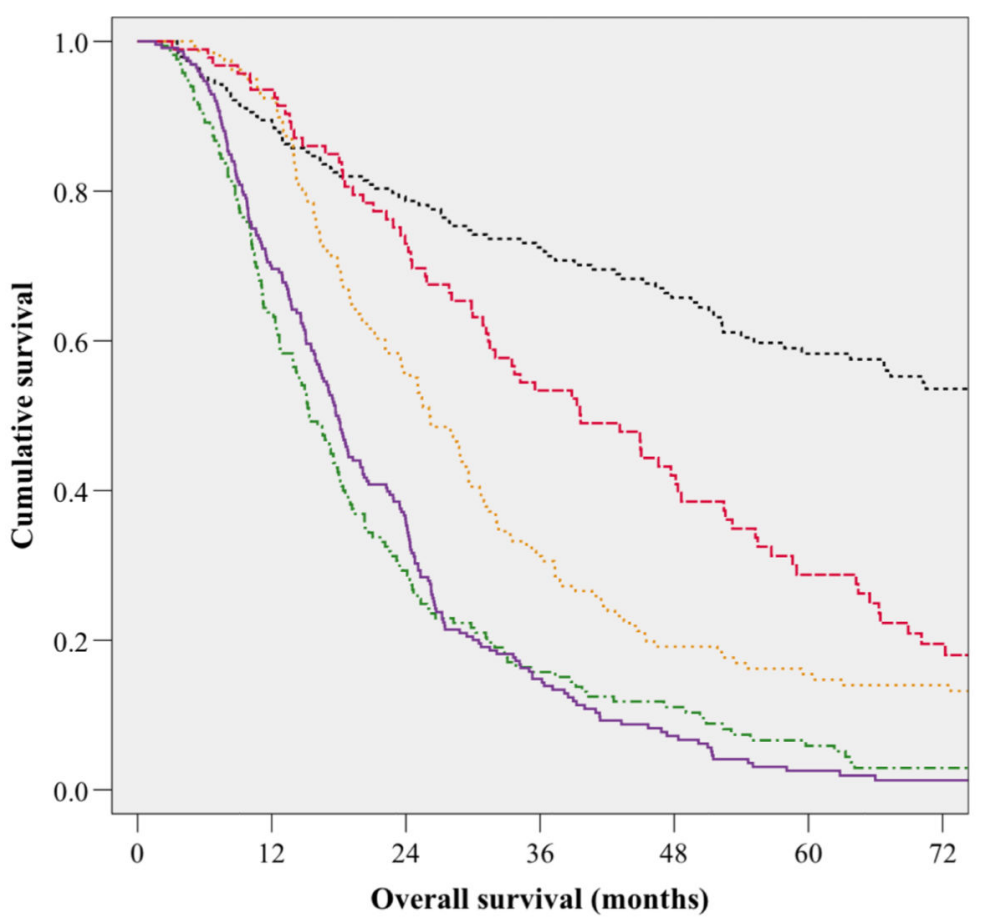

\begin{tabular}{|c|c|c|c|c|c|c|c|}
\hline $\begin{array}{l}\text { Recurrence } \\
\text { pattern }\end{array}$ & $\begin{array}{l}\text { No } \\
\text { recurrence }\end{array}$ & $\begin{array}{l}\text { Lung- } \\
\text { only }\end{array}$ & $\begin{array}{l}\text { Local- } \\
\text { only }\end{array}$ & Other & $\begin{array}{l}\text { Multiple- } \\
\text { site }\end{array}$ & $\begin{array}{l}\text { Liver- } \\
\text { only }\end{array}$ & $\begin{array}{l}\text { Median } \mathrm{OS} \\
\text { months }(95 \% \mathrm{CI})\end{array}$ \\
\hline $\begin{array}{l}\text { No recurrence } \\
(n=215)\end{array}$ & & $<0.001$ & $<0.001$ & $<0.001$ & $<0.001$ & $<0.001$ & $83.9(59.3-108.6)$ \\
\hline $\begin{array}{l}\text { Lung-only } \\
(n=93)\end{array}$ & $<0.001$ & & 0.003 & 0.341 & $<0.001$ & $<0.001$ & $39.6(29.3-50.0)$ \\
\hline $\begin{array}{l}\text { Local-only } \\
(n=158)\end{array}$ & $<0.001$ & 0.003 & & 0.626 & $<0.001$ & $<0.001$ & $26.2(22.8-29.5)$ \\
\hline $\begin{array}{l}\text { Other } \\
(n=18)\end{array}$ & $<0.001$ & 0.341 & 0.626 & & 0.003 & 0.005 & $29.4(23.5-35.3)$ \\
\hline $\begin{array}{l}\text { Multiple-site } \\
(n=227)\end{array}$ & $<0.001$ & $<0.001$ & $<0.001$ & 0.003 & & 0.855 & $17.7(16.2-19.3)$ \\
\hline $\begin{array}{l}\text { Liver-only } \\
(n=166)\end{array}$ & $<0.001$ & $<0.001$ & $<0.001$ & 0.005 & 0.855 & & $15.4(13.1-17.6)$ \\
\hline
\end{tabular}

FIG. 2.

Kaplan-Meier curves showing OS for different recurrence patterns with pairwise comparison of OS using the log-rank test. For clarity, the curve for 'other' recurrence has been omitted

Ann Surg Oncol. Author manuscript; available in PMC 2018 November 07.
Location of First

Recurrence

.:No recurrence

Local-only

- Liver-only

I'Lung-only

$\neg$ Multiple-site
. 


\section{롱 \\ 길}

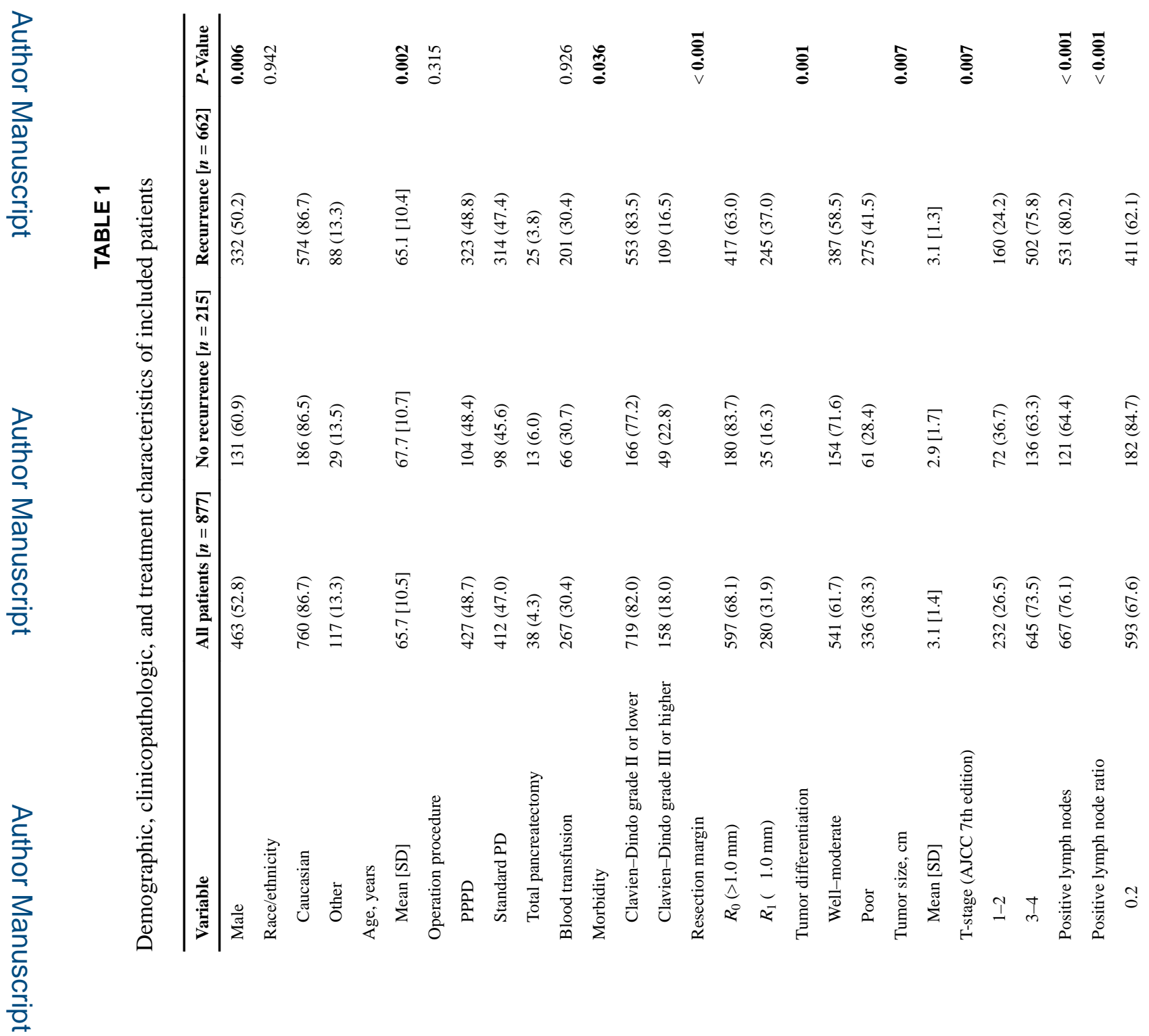
Ann Surg Oncol. Author manuscript; available in PMC 2018 November 07. 


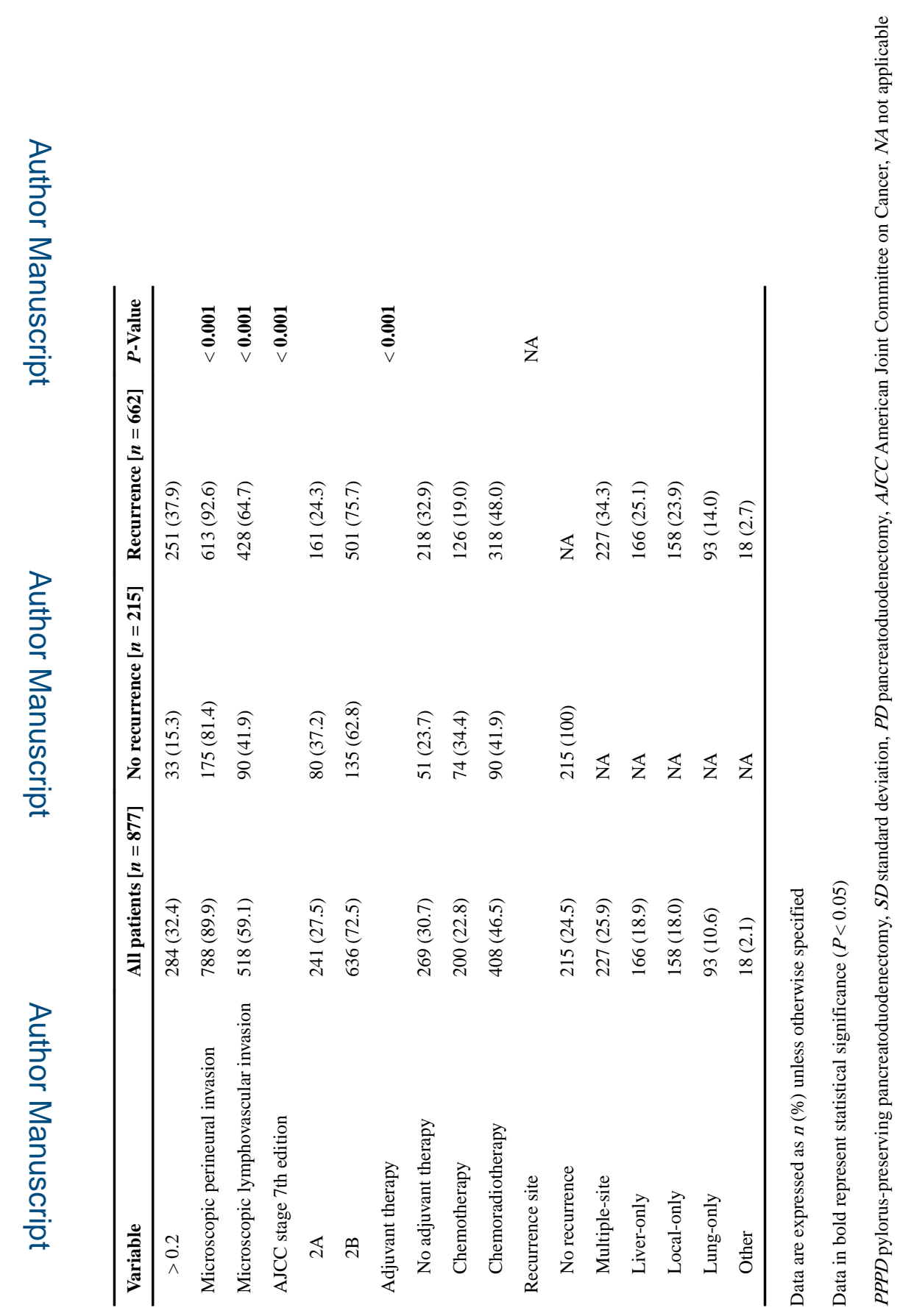

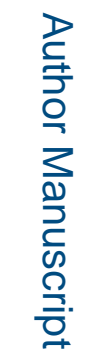




\section{롤 \\ }

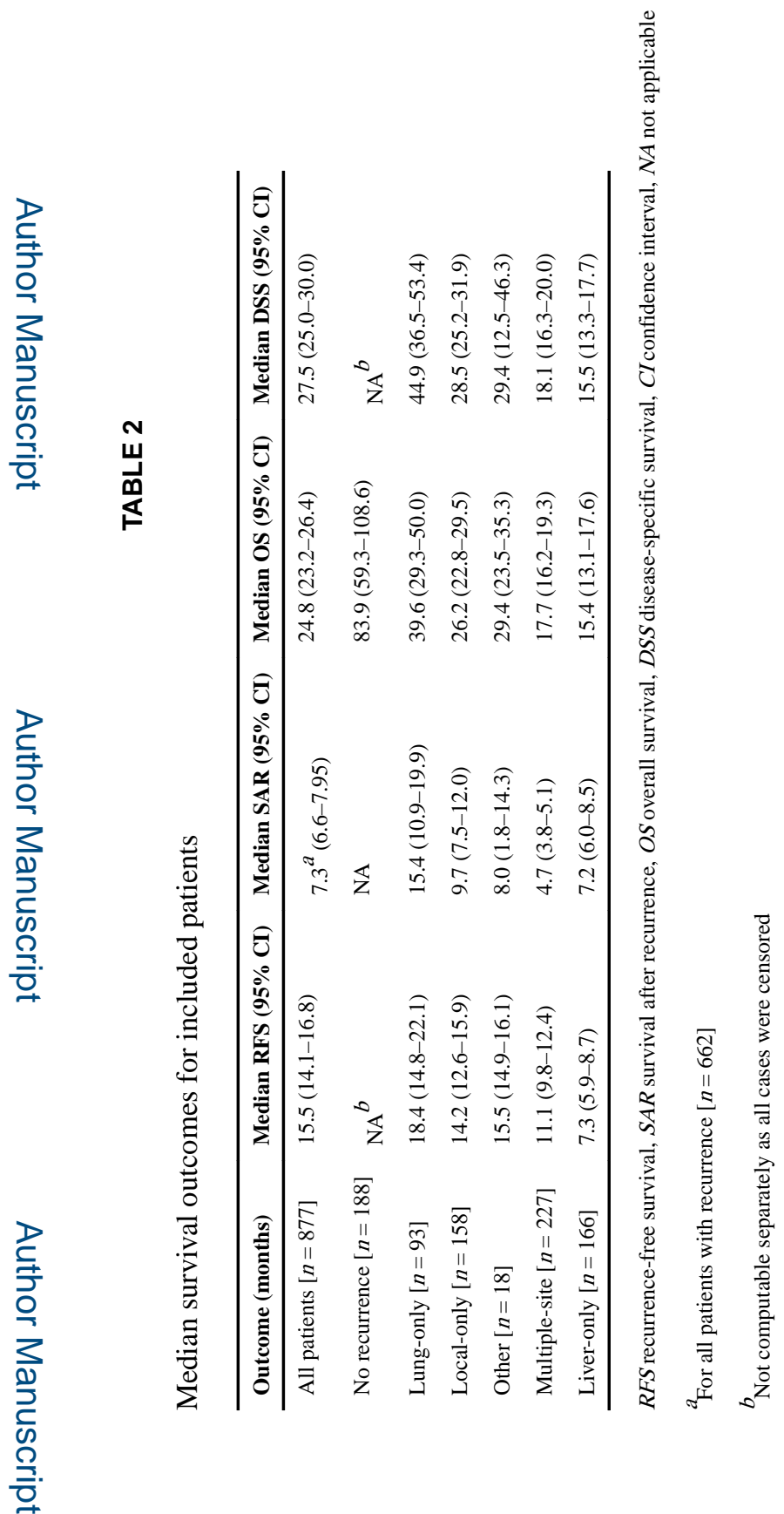

Ann Surg Oncol. Author manuscript; available in PMC 2018 November 07. 


\section{TABLE 3}

Multivariable Cox regression analysis for overall survival with recurrence locations as fixed covariates

\begin{tabular}{|c|c|c|c|}
\hline \multirow[t]{2}{*}{ Clinical characteristics } & \multicolumn{3}{|c|}{ Multivariable analysis } \\
\hline & HR & $95 \% \mathrm{CI}$ & $P$-Value \\
\hline Age at surgery, years ${ }^{a}$ & 1.01 & $1.01-1.02$ & $<0.001$ \\
\hline \multicolumn{4}{|l|}{ Complications ${ }^{b}$} \\
\hline Clavien $\geq 3$ versus Clavien $\leq 2$ & 1.22 & $1.00-1.50$ & 0.047 \\
\hline Tumor size, $\mathrm{cm}^{a}$ & 1.11 & $1.05-1.17$ & $<0.001$ \\
\hline \multicolumn{4}{|l|}{ Tumor differentiation grade } \\
\hline Poor vs. well/moderate & 1.24 & $1.06-1.45$ & 0.006 \\
\hline \multicolumn{4}{|l|}{ Microscopic perineural invasion } \\
\hline Yes versus no & 1.24 & $0.93-1.66$ & 0.147 \\
\hline \multicolumn{4}{|l|}{ Positive lymph node ratio } \\
\hline$>0.2$ versus $\leq 0.2$ & 1.49 & $1.26-1.76$ & $<0.001$ \\
\hline \multicolumn{4}{|l|}{ Resection margin status } \\
\hline$R 1$ versus $R 0$ & 1.37 & $1.16-1.61$ & $<0.001$ \\
\hline \multicolumn{4}{|l|}{ Adjuvant therapy } \\
\hline Adjuvant versus no adjuvant & 0.61 & $0.52-0.72$ & $<0.001$ \\
\hline \multicolumn{4}{|l|}{ Recurrence site } \\
\hline Multiple-site versus no recurrence & 4.78 & $3.65-6.25$ & $<0.001$ \\
\hline Liver-only versus no recurrence & 5.75 & 4.39-7.55 & $<0.001$ \\
\hline Local-only versus no recurrence & 2.46 & $1.86-3.24$ & $<0.001$ \\
\hline Lung-only versus no recurrence & 1.90 & $1.39-2.60$ & $<0.001$ \\
\hline Other versus no recurrence & 2.37 & $1.38-4.08$ & 0.002 \\
\hline
\end{tabular}

Significant values on multivariable analysis are shown in bold $(P<0.05)$

$H R$ hazard ratio, $C I$ confidence interval

${ }^{a}$ Continuous

${ }^{b}$ According to the Clavien-Dindo classification 
TABLE 4

Multivariable Cox regression analysis for overall survival using a landmark set at 12 months post-surgery

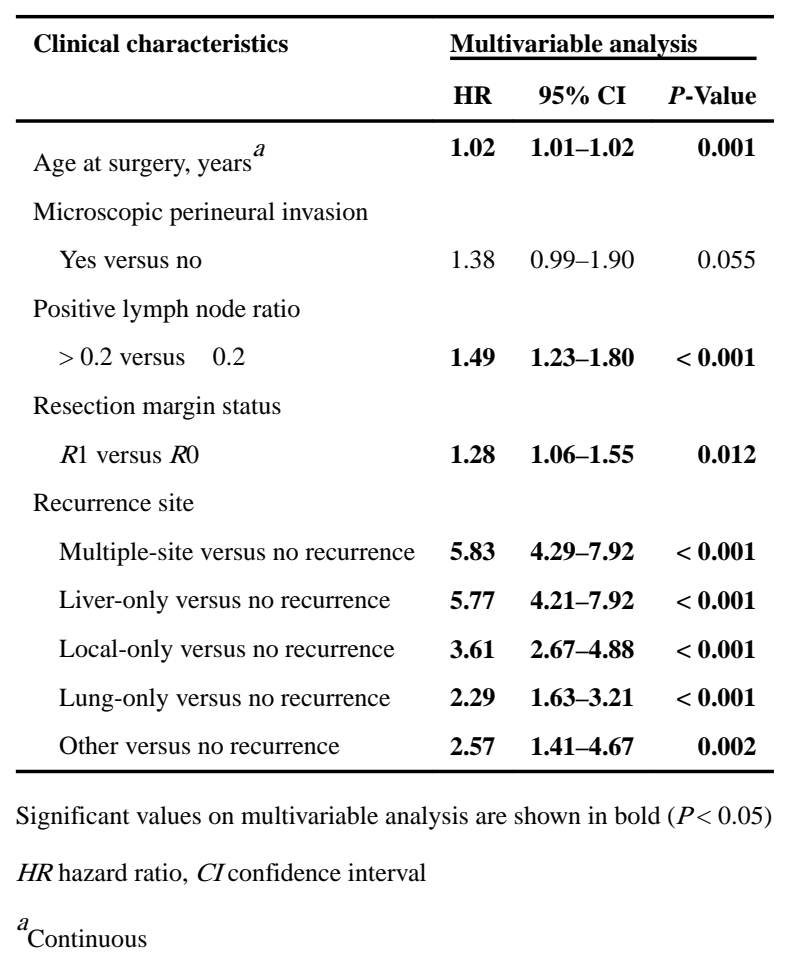

